\section{Commentary: A chess game for mesothelioma treatment: Not checkmate yet!}

\author{
Harvey I. Pass, MD
}

The pace for improving mesothelioma (MPM) survival compared with lung cancer reminds me of the "tortoise and the hare" race. Nevertheless, optimism for check point inhibition with (or without) chemotherapy is reflected in the excellent summary by Ripley and colleagues. ${ }^{1}$ Yes, Checkmate 743 is a legendary trial but as I read the Checkmate 743 manuscript in The Lancet, ${ }^{2}$ the following excerpt blows me away.

\section{"Better characterisation ...using transcriptomic and epigenetic profiling should guide ... therapeutic strategies, and aid in the identification of novel biomarkers.”}

What an understatement! Ripley and colleagues ${ }^{1}$ stress present/future immunotherapy protocols in their excellent discussion, but we are still in the infancy of understanding mechanisms that will help us figure out the best systemic therapy for a given MPM patient. Checkmate 743 is not the first large randomized nivolumab with ipilimumab (IPI/Nivo) trial versus chemotherapy for thoracic malignancies. ${ }^{3,4}$ In CheckMate 743 , the median overall survival was 17.1 months with IPI/Nivo versus 13.9 months with chemotherapy and "overall survival outcomes were similar in the subgroups with less than $1 \%$ and with $1 \%$ or higher [programmed death ligand 1] PD-L1 expressions." In CheckMate $227,{ }^{3}$ dual immunotherapy without chemotherapy for unresectable lung cancer also showed longer overall survival regardless of patients' PD-L1 expression levels. In non-small cell lung cancer,

\footnotetext{
From the Department of Cardiothoracic Surgery, NYU Langone Health, New York, NY.

Disclosures: The author reported advisory boards for Genentech/Roche.

The Journal policy requires editors and reviewers to disclose conflicts of interest and to decline handling or reviewing manuscripts for which they may have a conflict of interest. The editors and reviewers of this article have no conflicts of interest.

Received for publication Feb 2, 2022; accepted for publication Feb 2, 2022; available ahead of print Feb 9, 2022.

Address for reprints: Harvey I. Pass, MD, Department of Cardiothoracic Surgery, NYU Langone Medical Center, 530 First Ave, 9V, New York, NY 10016 (E-mail: harvey.pass@nyumc.org).

J Thorac Cardiovasc Surg 2023;165:369-70 $0022-5223 / \$ 36.00$

Copyright (C) 2022 Published by Elsevier Inc. on behalf of The American Association for Thoracic Surgery

https://doi.org/10.1016/j.jtcvs.2022.02.003
}

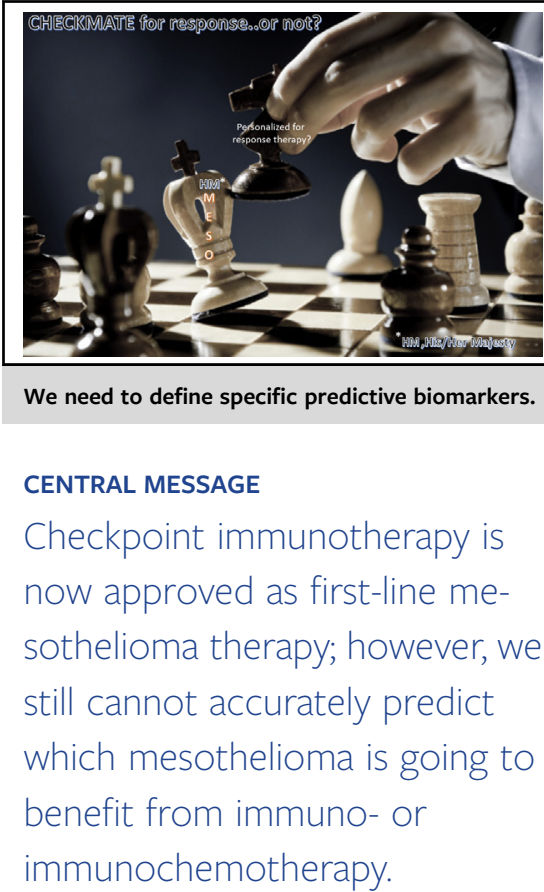

however, quantification of variants (ie, tumor mutation burden $[\mathrm{TMB}]$ ) was reported to be high in those having the benefit of IPI/Nivo. Because the median survival for IPI/Nivo in 743 were identical for epithelioid and nonepithelioid cases, does this mean that TMB differences between MPM histologies indeed do not exist except in rare cases as pointed out by the MPM Cancer Genome Atlas? ${ }^{5}$ We will have to wait for the TMA results from Checkmate 743 in the future; however, a fascinating "hot off the press" publication from one of these authors (A.M.) found a relationship between tumor junction burden and antigen presenation which predicted overall survival in patients treated with dual or single check point inhibitors. ${ }^{6}$ To confuse us further, previously untreated, unresectable MPM treated with durvalumab with platinum-pemetrexed responding to chemoimmunotherapy in the PrE0505 trial ${ }^{1,7}$ had a 20.4-month median survival (especially epithelioid tumors), and had higher nonsynonymous missense TMB and a more clonal mutation repertoire than nonresponding tumors! Additionally, responding tumors had specific alterations associated with immunogenic mutations proven with in vitro $\mathrm{T}$ cell clonal expansion techniques. ${ }^{7}$ So it might not be just the number of variants involved but the type of variant (and whether it's in the germline ${ }^{8-11}$ ), which influences whether the response is seen with check point inhibition. 
Other reasons for immunotherapy benefit in MPM? Burt and colleagues ${ }^{12}$ reported increases in B cells/tertiary lymphoid structure (B-cell follicles and germinal centers surrounded by a T-cell region) in resected MPM tumors after IPI/Nivo induction at the 2021 virtual AATS meeting. Tumor antigen with innate immune co-stimulation B-cell activation causes tumor responses either through T-cell neoantigen recognition or through enhanced antibody dependent cell death via immunoglobulin $\mathrm{M}$ antibodies... intriguing!

So, the clinical takeaway from 743 is that we now might have a first-line therapy for nonepithelioid MPM. Additionally, the recently published results at the three year followup of Checkmate 743.reveal continued benenfit of IPI/Nivo for MPM with a 3-year OS rates were $23 \%$ versus $15 \%$ for the combination compared with chemotherapy ${ }^{13}$ However, the real fun for thoracic oncology scientists (like every single one of the coauthors on The Journal of Thoracic and Cardiovascular Surgery report that this commentary blushes to pontificate on) will be to explore mechanism, biomarkers, and stratification for the right combination therapy for the right MPM patient.

\section{References}

1. Ripley RT, Mansfield AS, Sepesi B, Bueno R, Burt BM. Checkpoint blockade in unresectable pleural mesothelioma: event horizon for multimodal therapy. $J$ Thorac Cardiovasc Surg. 2023;165:364-8.

2. Baas P, Scherpereel A, Nowak AK, Fujimoto N, Peters S, Tsao AS, et al. Firstline nivolumab plus ipilimumab in unresectable malignant pleural mesothelioma (CheckMate 743): a multicentre, randomised, open-label, phase 3 trial. Lancet. 2021;397:375-86.
3. Hellmann MD, Ciuleanu TE, Pluzanski A, Lee JS, Otterson GA, AudigierValette C, et al. Nivolumab plus ipilimumab in lung cancer with a high tumor mutational burden. N Engl J Med. 2018;378:2093-104.

4. Cascone T, William WN Jr, Weissferdt A, Leung CH, Lin HY, Pataer A, et al. Neoadjuvant nivolumab or nivolumab plus ipilimumab in operable non-small cell lung cancer: the phase 2 randomized NEOSTAR trial. Nat Med. 2021;27: 504-14.

5. Hmeljak J, Sanchez-Vega F, Hoadley KA, Shih J, Stewart C, Heiman D, et al. Integrative molecular characterization of malignant pleural mesothelioma. Cancer Discov. 2018;8:1548-65.

6. Kosari F, Disselhorst M, Yin J, Peikert T, Udell J, Johnson S, et al. Tumor junction burden and antigen presentation as predictors of survival in mesothelioma treated with immune checkpoint inhibitors. J Thorac Oncol. 2022;17:446-54. https: //doi.org/10.1016/j.jtho.2021.10.022

7. Forde PM, Anagnostou V, Sun Z, Dahlberg SE, Kindler HL, Niknafs N, et al. Durvalumab with platinum-pemetrexed for unresectable pleural mesothelioma: survival, genomic and immunologic analyses from the phase 2 PrE0505 trial. Nat Med. 2021;27:1910-20.

8. Guo R, DuBoff M, Jayakumaran G, Kris MG, Ladanyi M, Robson ME, et al. Novel germline mutations in DNA damage repair in patients with malignant pleural mesotheliomas. J Thorac Oncol. 2020;15:655-60.

9. Bononi A, Goto K, Ak G, Yoshikawa Y, Emi M, Pastorino S, et al. Heterozygous germline BLM mutations increase susceptibility to asbestos and mesothelioma. Proc Natl Acad Sci U S A. 2020;117:33466-73.

10. Pastorino S, Yoshikawa Y, Pass HI, Emi M, Nasu M, Pagano I, et al. A subset of mesotheliomas with improved survival occurring in carriers of bap 1 and other germline mutations. J Clin Oncol. 2018;36:JCO2018790352.

11. Hassan R, Morrow B, Thomas A, Walsh T, Lee MK, Gulsuner S, et al. Inherited predisposition to malignant mesothelioma and overall survival following platinum chemotherapy. Proc Natl Acad Sci U S A. 2019;116:9008-13.

12. Burt B, Ramineni M, Jang H, Ramos D, Splawn T, Espinoza M, et al. A randomized trial of neoadjuvant PD-L1 versus PD-L1 plus CTLA-4 blockade in patients with malignant pleural mesothelioma. Presented at: American Association for Thoracic Surgery 101st Annual Meeting; April 30-May 2, 2021 (virtual meeting).

13. Peters S, Scherpereel A, Cornelissen R, Oulkhouir Y, Greillier L, Kaplan MA. First-line nivolumab plus ipilimumab versus chemotherapy in patients with unresectable malignant pleural mesothelioma: 3-year outcomes from CheckMate 743. Ann Oncol. 2022 Feb 3. https://doi.org/10.1016/j.annonc.2022.01.074 